\title{
Multifunktionale Bauteile aus Carbonbeton - Integrierte textilbasierte Sensorlösungen zur In-Situ- Strukturüberwachung adaptiver Gebäudehüllen
}

\author{
Eric Häntzsche ${ }^{1}$, Andreas Nocke ${ }^{1}$, Chokri Cherif ${ }^{1}$, Tobias Schuster ${ }^{2}$, Niels Neumann ${ }^{2}$, Dirk \\ Plettemeier $^{2}$, Michaela Gorges ${ }^{3}$, Marko Butler ${ }^{3}$, Viktor Mechtcherine ${ }^{3}$, Gert Schönfelder ${ }^{4}$ \\ Technische Universität Dresden, 01062 Dresden: \\ 1 ...Institut für Textilmaschinen und Textile Hochleistungswerkstofftechnik (ITM), \\ ${ }^{2}$...Institut für Nachrichtentechnik - Lehrstuhl Hochfrequenztechnik (IfN), \\ 3 ...Institut für Baustoffe (IfB). \\ ${ }^{4}$...Prignitz Mikrosystemtechnik GmbH, Margarethenstr. 61, 19322 Wittenberge
}

\section{Zusammenfassung}

In dem im Zuge der Initiative Zwanzig20 geförderten BMBF-Verbundprojekt Carbon Concrete Composite $C^{3}$ werden multifunktionale Betonbauteile mit innovativer Carbonbewehrungsstruktur für funktionalisierte Gebäudehüllen realisiert. Zur kontinuierlichen In-Situ-Strukturüberwachung werden diese neuartigen Textilbetonbauteile im Teilvorhaben $C^{3}$ Sense mit textilen Sensornetzwerken, bestehend aus piezoresistiven Carbonfaser-Sensoren (CFS) und faseroptischen Sensoren (FOS), ausgerüstet, die als integraler Bestandteil der aus Carbonfaser-Gelegestrukturen aufgebauten Betonbewehrung simultan bei der Flächenbildung durch textiltechnologische Fertigungsverfahren integriert werden. Mit diesen In-Situ-Sensoren wird eine Strukturüberwachung der Gebäudehülle hinsichtlich strukturmechanischer Veränderungen, die durch Deformationen und Risse oder infolge physikalischer Änderungen im Bauteilinneren durch mechanische, thermische oder klimatische Beanspruchungen hervorgerufen werden können, ermöglicht.

Keywords: Textilbeton, Carbonfaser, faseroptischer Sensor, resistiver Fasersensor, In-Situ-Sensorik

\section{Textilbeton - Carbon Concrete Composite $\mathrm{C}^{3}$}

Der seit 1995 an der Technischen Universität Dresden (SFB 528) und der RheinischWestfälischen-Hochschule Aachen (SFB 532) entwickelte Textilbeton stellt eine neuartige Form von Betonverbundkonstruktion dar, deren Bewehrungsstruktur im Gegensatz zu konventionellen Stahlbewehrungen aus nichtkorrosionsanfälligen Glas- oder Carbonfasern (CF) hergestellten textilen Verstärkungshalbzeugen besteht, über die der Lastabtrag realisiert wird. Damit wird die geringe Zugfestigkeit $R_{M, C^{3}}$ des Betons kompensiert. Anders als bei klassischen Stahlbewehrungen $\left(R_{M, S t}<0,64 \mathrm{GPa}\right)$ sind aufgrund der höheren Zugfestigkeit von CF $\left(R_{M, C F}=4,0 \mathrm{GPa}\right)$ in Kombination mit der viermal kleineren Dichte wesentlich leichtere Betonbauteile mit geringeren Wandstärken herstellbar. Das Gesamtgewicht kann zudem durch eine Reduzierung der sonst im Stahlbetonbau übliche Betondeckung nach DIN 1045-1 bei Nutzung inerter Bewehrungsmaterialien wie CF signifikant vermindert werden. Das unter der Bezeichnung TUDALIT ${ }^{\circledR}$ (DIBt Zul.-Nr.: Z31.10-182) verfügbare Verstärkungshalbzeug aus CF-Roving für die Herstellung von leichten $C^{3}$-Bauteilen (Abb. 1) besteht aus einem offenen biaxial-verstärkten gitterartigen Gelege in $0^{\circ} / 90^{\circ}$-Anordnung.

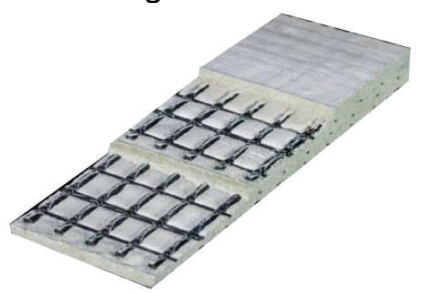

Abb. 1: Mehrschichtiges Carbon Concrete Composite $\left(C^{3}\right)$-Bauteil mit Bewehrungsstruktur aus Carbonfaser-Biaxialgelege

Das in $0^{\circ}$-Richtung und damit in der Hauptbelastungsrichtung orientierte Verstärkungsfadensystem besteht aus CF-Roving mit einer Feinheit von $3,3 \cdot 10^{-3} \mathrm{~kg} \cdot \mathrm{m}^{-1}$ und 50.000 Einzelfilamenten (50k), der in $90^{\circ}$ Richtung ausgerichtete CF-Roving mit 12.000 Einzelfilamenten weist dagegen nur eine 
Feinheit von $0,8 \cdot 10^{-3} \mathrm{~kg} \cdot \mathrm{m}^{-1}$ auf, da Querkontraktionen bei reiner Zugbeanspruchung allein durch die Druckfestigkeit $\left(R_{D}=0,05 \ldots 0,09 \mathrm{GPa}\right)$ des Betons abgefangen werden können. Die orthogonal mit fester Gitteröffnung von 10,7 mmx14,3 mm zueinander angeordneten Fadensysteme werden maschinelle auf einer MultiaxialKettenwirkmaschine bis zu Breiten von max. $1,25 \mathrm{~m}$ gefertigt und durch ein zusätzliches Bindefadensystem kraftschlüssig und verschiebefest gegeneinander fixiert (Abb. 2).

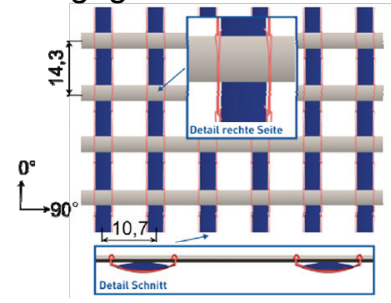

Abb. 2: Schematischer Aufbau $C^{3}$-Bauteilbewehrung aus CF-Biaxialgelege

Für die Verbesserung der Handhabbarkeit während der Einbringungen in den Beton wird das derart hergestellte Biaxial-Gelege mit einer Styrol-Butadien-Kautschuk (SBR)-Dispersion durch Inline-Foulardierung beschichtet und vor dem Aufwickeln in einem nachgeschalteten IRHeizfeld getrocknet. In Abhängigkeit vom Beschichtungsgrad sind Flächengewichte bis $490 \mathrm{~g} / \mathrm{m}^{2}$ realisierbar, die anschließend ohne weitere Vorbereitung mit Feinbeton TUDALITTF10-PAGEL im Handlaminier- oder Gießverfahren zu $\mathrm{C}^{3}$-Bauteilen verarbeitet werden können, die nach 28 Tagen vollständig ausgehärtet sind. Die (Zug-)Kraftübertragung vom Beton auf die lastabtragende textile Bewehrung erfolgt formschlüssig über eine stoffschlüssige Verankerung des Betons durch die Gitteröffnungen des CF-Biaxialgeleges.

\section{Sensorintegration in Betonbewehrungen aus Carbonfasergelegen}

Als erstes Messprinzip wird der Ansatz der Dehnungsmessung mittels piezoresistiver CFSensoren (CFS) verfolgt. Das zweite Messprinzip basiert auf der Dehnungsmessung mittels faseroptischer Sensoren (FOS) mit laserinduzierten Fasergittern (Bragg- und langperiodische Gitter). Beide Sensormaterialien werden als ein- bzw. zweidimensional ebene und integral-messende Dehnungssensoren bei der Flächenbildung mittels Kettenwirkmaschine MALIMO 14024 (Abb. 3) mit der später im $C^{3}$-Betonverbund lastaufnehmenden und zuvor beschriebenen CF-Gelegestruktur aus Carbonfaser kraftschlüssig maschinell miteinander verbunden. Die Ankopplung beider
Sensortypen an die in später lastabtragende Betonbewehrungsstruktur erfolgt kontinuierlich über ein Polyester-Bindefadensystem aller $4,0 \mathrm{~mm}$, dass sowohl Sensorfaden und die in $0^{\circ} / 90^{\circ}$-Orientierung verlaufenden CFVerstärkungsfadensysteme miteinander verbindet (vgl. Abb. 2). Bei eindimensionaler Sensorform, z. B. direkter Ablage des Sensors auf dem in $0^{\circ}$-Richtung verlaufenden CFKettfadensystem ist dabei der über die Anzahl der Bindungspunkte generierte Kraftschluss entscheidend für ein beanspruchungskorrelatives Abbildungsvermögen beider Sensortypen. Bei zweidimensionalen Sensorlayouts, z. B. mäandrierende oder ondulierende Integration des Fasersensors über mehrere in $0^{\circ}$-Richtung verlaufende CFRoving der Gelegestruktur bzw. um mehrere Gitteröffnungen, kann theoretisch ein zusätzlicher Formschluss zur Verbesserung der Anbindung der Sensoren an den $\mathrm{C}^{3}$ Verbund erreicht werden.

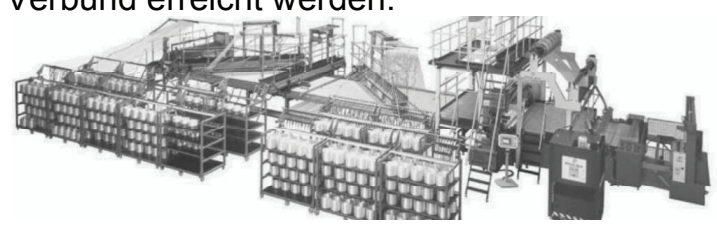

Abb. 3: MALIMO 14024 Kettenwirkmaschine zur Fertigung von CF-Biaxialgelegen und simultaner Sensorintegration bei der Flächenbildung [Quelle: Karl Mayer Textilmaschinenfabrik $\mathrm{GmbH}]$

\section{Resistive Fasersensoren}

Bei der Umsetzung zweidimensionaler resistiver Dehnungssensorlayouts, vergleichbar mit der Gestalt von DMS-Messgittern, werden zwei Ansätze verfolgt. Ersterer (Abb. 4) sieht die Nutzung des intrinsisch leitfähigen CF-Fadenmaterials der in $0^{\circ} / 90^{\circ}$ verlaufenden Bewehrungsstruktur selbst unter Ausnutzung des piezo-resistiven Effektes zur Dehnungsmessung vor [1]-[2]. Dafür ist kein zusätzliches Sensormaterial erforderlich. Der zweite Ansatz verfolgt die Integration zusätzlicher CF-Rovings in $0^{\circ}$ - und $90^{\circ}$ Richtung, die jedoch eine signifikant geringere Feinheit $\left(6,7 \cdot 10^{-5} \mathrm{~kg} \cdot \mathrm{m}^{-1}\right)$ und nur 1.000 Einzelfilamente $(1 \mathrm{k})$ aufweisen. Frühere Untersuchungen haben gezeigt, dass als Dehnungssensor fungierende 1k-CF-Rovings ein besseres Übertraguns- bzw. Abbildungsverhalten aufweisen [3].

Für die Realisierung beider Ansätze sind aufgrund der elektrischen Leitfähigkeit der CF $\rho^{-1}=(0,15 \ldots 0,17) \mathrm{S} \cdot \mathrm{m}^{-1}$, die sowohl Sensorals auch Bewehrungsstruktur bilden, Isolationsmaßnahmen zwingend erforderlich, um tatsächlich nur lokal die über den 
Integrationspfad der resistiven Fasersensoren akkumulierte Dehnung als Widerstandsänderung zu erfassen, die aus beanspruchungsinduzierter Geometrie- und Leitfähigkeitsänderung resultiert.

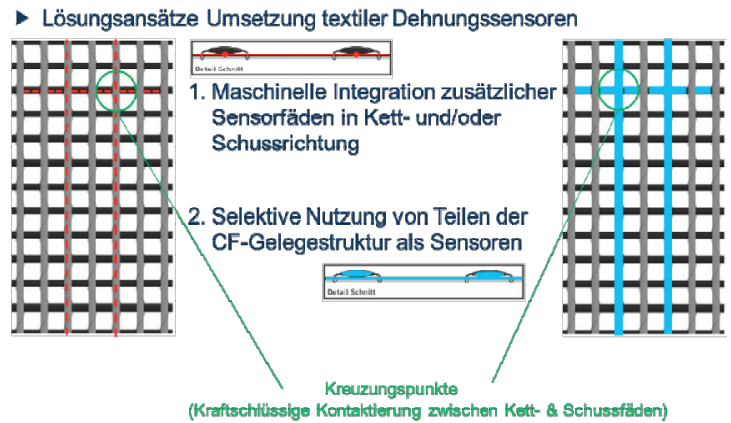

Abb. 4: Realisierung diversitärer Sensorlayouts: Integration zusätzlicher CFRovings (li.) oder selektive Nutzung definierter Pfade innerhalb des CFBiaxialgestricks (re.)

Es werden dabei sowohl filmbildende Polymerbeschichtungen (SBR und Polyvinylchlorid PVC-P) als auch im Friktionsspinnverfahren aus Polypropylen (PP)-Stapelfasern mit $30 \mathrm{~mm}$ Faserlänge gesponnene Isolationsummantelungen hergestellt und anschließend maschinell in die CF-Gelege eingearbeitet. Im Bereich der Kreuzungspunkte der zum Aufbau zweidimensionaler Sensorlayouts benötigten Teilstücken aus CF-Roving wird die Isolation partiell entfernt, durch Leitkleberapplikation der elektrische Kontakt gesichert und anschließend die Isolation wiederhergestellt.

\section{Sensorcharakteristik von CFS im $\mathrm{C}^{3}$}

Gegenstand erster Untersuchungen stellen Zugversuche zum elektro-mechanischen Verhalten eindimensionaler und in der $0^{\circ}$ - oder Hauptbelastungsrichtung orientierten Sensoren aus isoliertem 1k-CF-Roving in $\mathrm{C}^{3}$ Probenkörpern (vgl. Abb. 5) mit den Dimensionen $[\mathrm{LxBxT}]=500 \times 100 \times 12 \mathrm{~mm}^{3}$ dar. Diese sind durch drei äquidistant zueinander angeordnete Lagen aus CF-Biaxialgelege verstärkt. Die mittig angeordnet Lage fungiert als Sensorträger, die beiden dazu symmetrisch angeordneten Lagen ohne Sensoren dienen der zusätzlichen Verstärkung.

24 Stunden nach der Betonierung erfolgt die Ausschalung aus der Gussform. Nach einer Aushärtung über 28 Tage bei Lagerbedingungen nach DIN 18555-3 wird die Zugprüfung mit fünf kraftgeregelten Belastungszyklen bis ca. $0,39 \%$ Dehnung mit einer Dehnrate von $0,5 \mathrm{~mm} \cdot \mathrm{min}^{-1}$ und abschließend stetiger Beanspruchungssteigerung der $\mathrm{C}^{3}$-Probe bis zum Bruch durchgeführt.
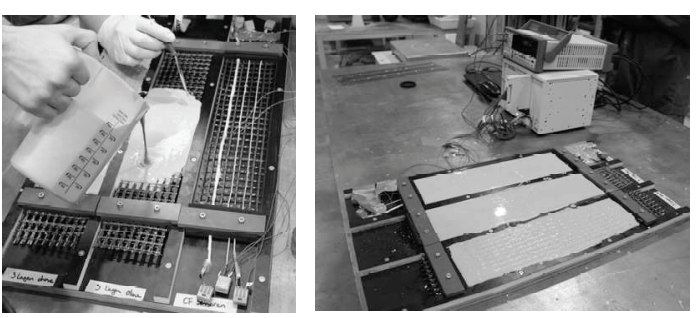

Abb. 5 Betonierung von $C^{3}$-Probenkörpern mit integrierter Sensorik zur Strukturüberwachung

Dieser tritt bei den realisierten Probenkörpern bei ca. $(740 \pm 50) \mathrm{MPa}$ Zugspannung und $(0,79 \pm 0,08) \%$ Dehnung ein. Risse der Betonmatrix sind bis ca. $250 \mathrm{MPa}$ im KraftDehnungsverlauf feststellbar. Bei größerer Zugbeanspruchung trägt ausschließlich die textile CF-Bewehrung, was dem realen Tragzustand bewehrter zugbeanspruchter Betonbauteile entspricht. Bei der Zugbelastung erfolgt die Erfassung des Sensorwiderstandes nach dem Zweileitermessverfahren mit einem Präzisions-Labormultimeter FLUKE 8846A. Die Abb. 6 zeigt exemplarisch den Signalgang eines im $\mathrm{C}^{3}$-Verbund integrierten hochohmigen und aus 1k-CF-Roving mit gesponnenem Fasermantel zur elektrischen Isolation bestehenden CFS (Grundwiderstand $\mathrm{R}_{0} \approx 300 \Omega$ ) bei zyklischer Zugbeanspruchung. Es ist eine gute Korrelation mit der am $\mathrm{C}^{3}$ Probenkörper anliegenden Zugbeanspruchung zu erkennen. Darüber zeigen die integrierten Sensoren eine ausgeprägte negative Drift über den Belastungszeitraum.

Die mittlere Empfindlichkeit k (Übertragungsfaktor oder k-Faktor) der untersuchten hochohmigen CF-Sensoren im $\mathrm{C}^{3}$ (vgl. Abb. 6) wird in ersten Versuchsreihen $\mathrm{zu} k \approx 0,18$ ermittelt.

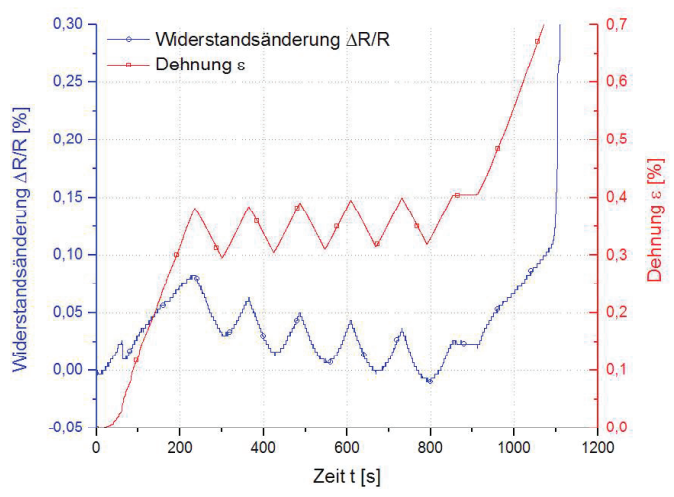

Abb. 6 Sensorcharakteristika eines hochohmigen eindimensionalen $1 \mathrm{k}-\mathrm{CF}$ Sensor im $C^{3}$-Verbund bei zyklischer Zugbeanspruchung

Beide Effekte (Drift und niedriger k-Faktor) sind vermutlich auf eine sukzessive Ausrichtung der Einzelfilamente des resistiven Fasersensors in 
der Hauptbeanspruchungsrichtung und/oder durch fraktionierten Auszug bzw. gleichzeitiges teleskopierendes Abgleiten einzelner Fasern aus bzw. an dem als Sensor fungierenden CFRoving und dadurch bedingter sukzessiver aber irreversibler Änderung des ohmschen Widerstandes (Sensorsignal) zurückzuführen. Gegenstand weiterer Untersuchungen umfassen die Charakterisierung der Widerholgenauigkeit integrierter CFS im $\mathrm{C}^{3}$ bei zyklischer Belastung, die Evaluierung der Langzeitstabilität bei hohen Lastwechselzahlen sowie der Einfluss der kraft- und zusätzlichen formschlüssigen Anbindung von CFS an die textile Bewehrung auf das Sensorverhalten.

\section{Faseroptische Sensoren}

Faseroptische Sensoren (FOS) sind äußerst kompakt sowie elektromagnetisch und chemisch unempfindlich. Sie eignen sich daher ideal zur In-Situ-Strukturüberwachung von Bauwerken. Fasergitter erlauben die Realisierung hochempfindlicher, lokalisierter FOS für Dehnung und Temperatur [3]. Wie in Abb. 7 am Beispiel eines Dehnungssensors gezeigt, wird dabei eine Änderung der Messgröße als eine Verschiebung der Fasergitterresonanz im Transmissions- oder Reflexionsspektrum des FOS nachgewiesen.

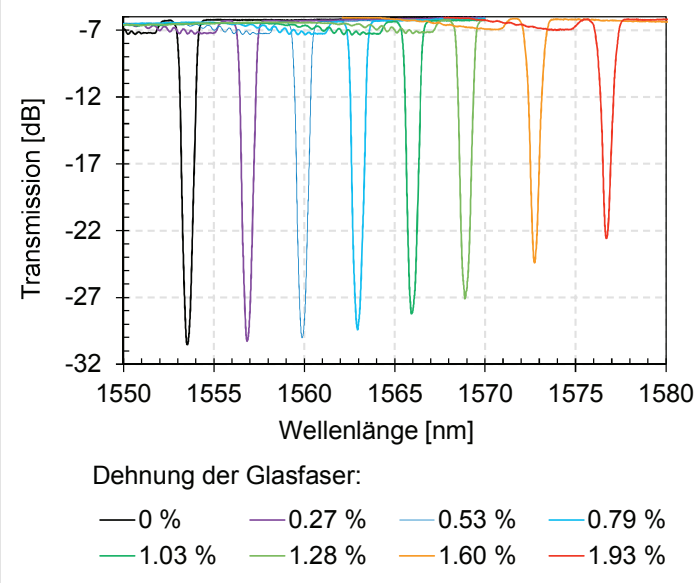

Abb. 7: Spektrale Verschiebung der Fasergitterresonanz durch Dehnung der optischen Faser

Solche Fasergittersensoren können auf die Oberflächen von Bauwerken aufgeklebt oder direkt in die Betonbauteile integriert werden. Im letzteren Fall ist eine Verkapselung (Packaging) der FOS und der faseroptischen Zuleitungen erforderlich, um optische Verluste und Störungen durch eine unkontrollierte mechanische Beanspruchung oder das unkontrollierte Eindringen von Feuchtigkeit zu verhindern. Etablierte Packaging-Konzepte, welche für die Integration in voluminöse stahlbewehrte Betonbauteile entwickelt wurden, können jedoch nicht in dünnen CFbewehrten Bauelementen untergebracht werden ohne eine signifikante Störung darzustellen. Ziel ist es daher Prozesse für die Integration von Fasergittersensoren in Carbonbeton-Bauteile und das entsprechende Packaging zu untersuchen, damit die FOS möglichst bündig und störungsfrei in die textile CF-Bewehrung integriert werden kann.

Es werden unterschiedliche FOS-Konzepte zur In-Situ-Strukturüberwachung entwickelt und in Funktionsmustern umgesetzt. Diese basieren nicht nur auf den in der Strukturüberwachung etablierten kurzperiodischen Faser-BraggGittern (FBG), sondern auch auf langperiodischen Fasergittern (LPG). Mit LPG können unterschiedliche Mantelmoden der optischen Faser genutzt und damit die Empfindlichkeit der Fasergitterresonanz für Temperatur und Dehnung sehr genau eingestellt werden. Die definierte Wechselwirkung zwischen dem evaneszenten Feld der Mantelmoden und dem Umgebungsmedium der optischen Faser ermöglicht außerdem neuartige Ansätze für $\mathrm{pH}-$ Wert- und Feuchtigkeitsmessungen [4].

\section{Integration der faseroptischen Sensoren in Carbonbeton-Funktionsmuster}

Die Integration der FOS in die CarbonbetonFunktionsmuster erfolgt in drei Schritten: Packaging, Anbindung an die textile CFBewehrung und Einbetonieren. Abb. 8 zeigt ein CF-Biaxialgelege mit integriertem FBG-Sensor vor der Einbetonierung. In Abb. 9 wird der prinzipielle Längsschnitt durch einen integrierten Fasergittersensor dargestellt.

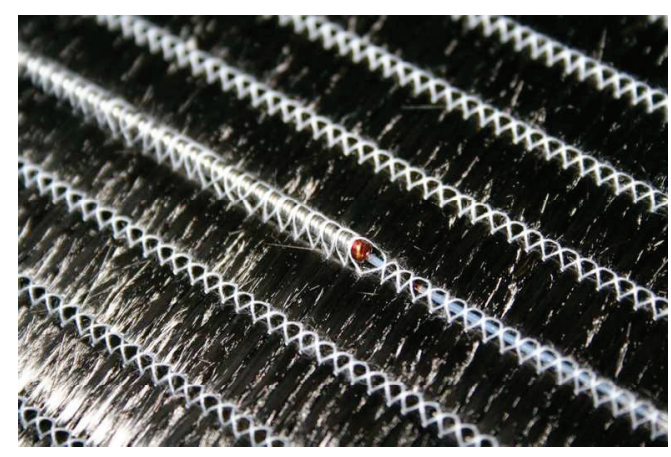

Abb. 8 CF-Biaxialgelege mit integriertem faseroptischen Sensor 


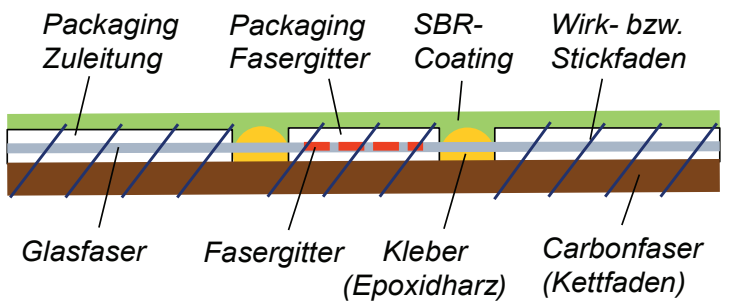

Abb. 9: Längsschnitt durch einen integrierten faseroptischen Sensor

Als Packaging für die Fasergitter und die faseroptischen Zuleitungen werden unterschiedliche Materialien untersucht. Zu Vergleichszwecken werden Experimente ohne zusätzliches Packaging durchgeführt, bei denen die optische Faser ausschließlich durch das bereits vorhandene dünne Acrylatcoating geschützt werden. Die verkapselten faseroptischen Sensoren werden maschinell mit einem Kettfaden der CF-Bewehrung verbunden. In Abhängigkeit des untersuchten Sensortyps wird die Glasfaser an der Grenze des Fasergittersensors entweder mit der CFBewehrung oder mit dem Packaging verklebt. Die, für die Verarbeitung der CF-Bewehrung erforderliche, Beschichtung mit SBR erfolgt nach der Anbindung des FOS. Die CF-Gelege mit den integrierten FOS werden auf die gleiche Weise wie die CF-basierten resistiven Sensoren einbetoniert. Die resultierenden Funktionsmuster werden 28 Tage getrocknet bevor die Sensitivität der integrierten Sensoren getestet wird.

\section{Sensorcharakteristik von Fasergittern im $\mathrm{C}^{3}$}

Die Transmissionsspektren der FOS werden nach jedem Integrationsschritt gemessen und mit dem Verhalten der unverkapselten Fasergitter verglichen. Dabei zeigt sich, dass FOS ohne Packaging ungeeignet für die Integration in Carbonbeton-Bauteile sind.

Durch die Schrumpfung des getrockneten Betons treten axiale und laterale Kräfte auf die optische Faser auf, welche zu Verlusten führten, die mit der Länge der faseroptischen Zuleitungen skalieren. Diese Verluste können durch das Packaging mit einem 0,8 mm dicken PTFE-Schlauch deutlich reduziert werden.

Bei FBG-basierten Dehnungssensoren ohne Packaging wird eine unerwünschte Verbreitung der Fasergitterresonanz beobachtet, die aus ungleichmäßigen lateralen Kräften resultiert und die Empfindlichkeit der Sensorauswertung beeinträchtigt. Dieser Effekt kann durch das Packaging mit einem $0,8 \mathrm{~mm}$ dicken und $70 \mathrm{~mm}$ langen Edelstahlröhrchen (Innendurchmesser: $\quad 0,5 \mathrm{~mm}$ ) verhindert werden. Mit $20 \mathrm{~mm}$ langen Klebeverbindungen aus kalthärtendem Epoxidharz wird eine gute
Kraftübertragung zwischen optischer Faser und CF-Gelege erreicht. Abb. 10 zeigt das resultierende Sensorverhalten. Dargestellt werden die spektrale Verschiebung der Gitterresonanz und die prozentuale Dehnung des Carbonbetons in Abhängigkeit von der anliegenden Zugkraft. Nach der Rissbildung des Betons wird eine Sensitivität von $6,5 \mathrm{~nm} / \%$ reproduzierbar erreicht.

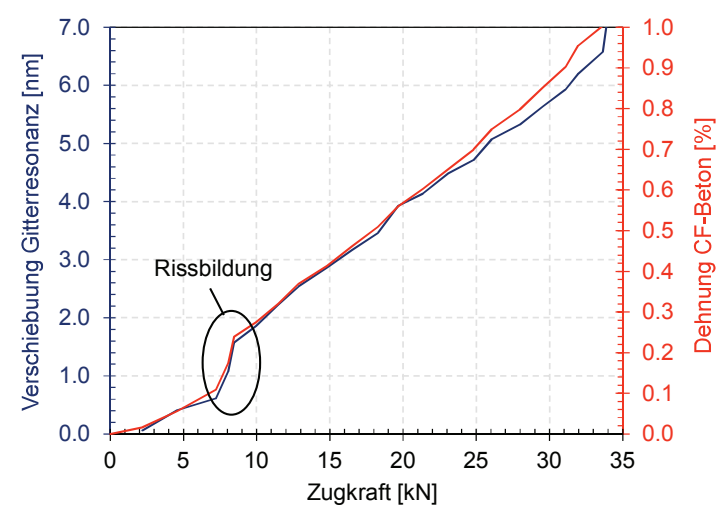

Abb. 10: Verschiebung der Gitterresonanz bei zunehmender Dehnung des CFBetonbauteils

Die LPG-basierten FOS sind äußerst empfindlich gegenüber Biegung und der Brechzahl des Umgebungsmediums. Die LPG werden daher mit heißhärtendem Epoxidharz freischwebend in halboffene, $2,0 \mathrm{~mm}$ dicke Glasröhrchen eingeklebt. Die Temperatursensoren werden zusätzlich mit Schrumpfschlauch geschützt. Auf diese Weise bleiben FOS und Packaging bei der Einbettung unversehrt und erreichen im gesamten Messbereich von $23^{\circ} \mathrm{C}$ bis $70^{\circ} \mathrm{C}$ eine Empfindlichkeit von $0.15 \mathrm{~nm} /{ }^{\circ} \mathrm{C}$.

Für die Realisierung der Feuchtesensoren werden die Glasröhrchen mit einem Hydrogel gefüllt, welches in einer feuchten Umgebung sein Volumen und seine Brechzahl ändert. Es kann nachgewiesen werden, dass dieser Vorgang zu einer signifikanten Verschiebung der Fasergitterresonanz führt. Zurzeit wird an einer feuchtedurchlässigen, textilen Ummantelung geforscht, die eine schädigungsfreie Einbettung in die Betonteile ermöglichen soll.

\section{Zusammenfassung}

Anhand von Zugversuchen an mit Gelegestrukturen aus Carbonfaser (CF) bewehrten Textilbetonprobenkörpern $\left(\mathrm{C}^{3}\right)$ und darin integrierten textilen Strukturüberwachungssystemen, bestehend aus resistiven CF-Fasersensoren und faseroptischen Sensoren, konnten grundlegende Erkenntnisse zu Sensor- und $\mathrm{C}^{3}$-Verbundwerkstoffcharakteristika gewonnen werden, die 
wesentlich für eine Verbesserung des Materialverständnisses von Textilbetonen beitragen werden.

Weiterhin wurden anwendungsangepasste faseroptische Sensoren (FOS) auf Basis von Fasergittern entwickelt. Es konnte experimentell gezeigt werden, dass das Packaging der optischen Faser ausschlaggebend für die Funktionalität der FOS ist.

Parallel zu den Forschungsaktivitäten erfolgen Entwicklungsarbeiten zu einer skalierbaren miniaturisierten und in den $\mathrm{C}^{3}$-Verbund integrierbaren Low-Power Elektronik, die energieautark zur messtechnischen Datenerfassung und -verarbeitung der integrierten faserbasierten Sensorik im späteren $\mathrm{C}^{3}$-Bauteilen genutzt werden soll.

\section{Danksagung}

Das diesem Bericht zugrundeliegende Vorhaben wird aus Mitteln des Bundesministeriums für Bildung und Forschung (BMBF) unter dem Förderkennzeichen 03ZZ0305P gefördert. Die Verantwortung für den Inhalt dieser Veröffentlichung liegt bei den Autoren.

\section{Literaturnachweis}

[1] OWSTON, C. N.: Electrical properties of single carbon fibres. Journal of Physics D: Applied Physics 3(1970)11, pp. 1615- 1626

[2] Häntzsche, E.; Matthes, A.; Nocke, A.; Cherif, Ch. Sensors and Actuators A: Physical A203(2013)1, pp. 189-203. -ISSN 0924-4247. DOI: 10.1016/j.sna.2013.08.045

[3] Haque, M.E.; Zain, M.F.M. et al.: Recent Application of Structural Civil Health Monitoring Using WSN and FBG. World Applied Sciences Journal 20 (2012) 4, pp. 585-590

[4] Schuster, T., Neumann, N., Plettemeier, D., Körbitz, R., \& Richter, A.: A fiber-optic pH sensor with wireless Radio over Fiber read-out. In SENSORS, 2015 IEEE, pp. 1-4 